\title{
Fluorescence Properties and Photophysics of Cy3 Linked Covalently to DNA
}

\author{
Matthew E. Sanborn ${ }^{\S}$, Brian K. Connolly ${ }^{\S}$, Kaushik Gurunathan ${ }^{\S}$ and Marcia Levitus ${ }^{\S \ddagger *}$ \\ ${ }^{\S}$ Department of Chemistry and Biochemistry, ${ }^{\dagger}$ Department of Physics and ${ }^{\star}$ The Biodesign Institute. \\ Arizona State University, Tempe, Arizona 85287-5601.
}

\section{Supporting Information}

\section{1) Pump Probe Transient Absorption Spectroscopy}

Pump-probe experiments use a light pump pulse to promote the sample to an excited state and then monitors the relaxation back to the ground state with a probe pulse. Experiments using a $610 \mathrm{~nm}$-probe pulse cause stimulated emission in the $\mathrm{Cy} 3$ emission band. As the excited molecules relax, the probability for stimulated emission decreases until the excited-state population is completely depleted. In this way, this decay measures the lifetime of the excited state directly.

\section{Experimental:}

The setup, described previously ${ }^{1}$ uses a $\mathrm{KHz}$ regeneratively amplified femtosecond Ti:Sa laser (Spectra Physics) and a pump-probe optical setup. The sample was contained in a $1 \mathrm{~mm}$ path length cuvette, and was excited at $515 \mathrm{~nm}$ with a 150 fs pump pulse. The probe beam is created by taking $10 \%$ of the pump beam and passing it through a sapphire plate to generate a white light continuum. An optical delay line was used to control the time delay between the excitation and the probe pulses in the few ps to 3 ns range. The probe and reference signals were collected by a spectrograph and recorded by a photodiode at a selected wavelength. Time-resolved fluorescence anisotropy was measured using the same setup and controlling the polarization of the probe pulse with a sheet polarizer. 


\section{Results:}

\section{$\underline{\text { Fluorescence lifetimes }}$}

Figure S1 shows the fluorescence decays obtained by pump-probe. The small differences between these results and those obtained by TCSPC are a consequence of the differences in the temperature of the laboratories where the experiments were performed. These results confirm the striking differences between the free dye and the Cy3-DNA conjugates, and between the single-stranded and duplex 5'modified samples. The signal was normalized and inverted for plotting purposes. Actual amplitudes were around -0.004 units of absorbance.

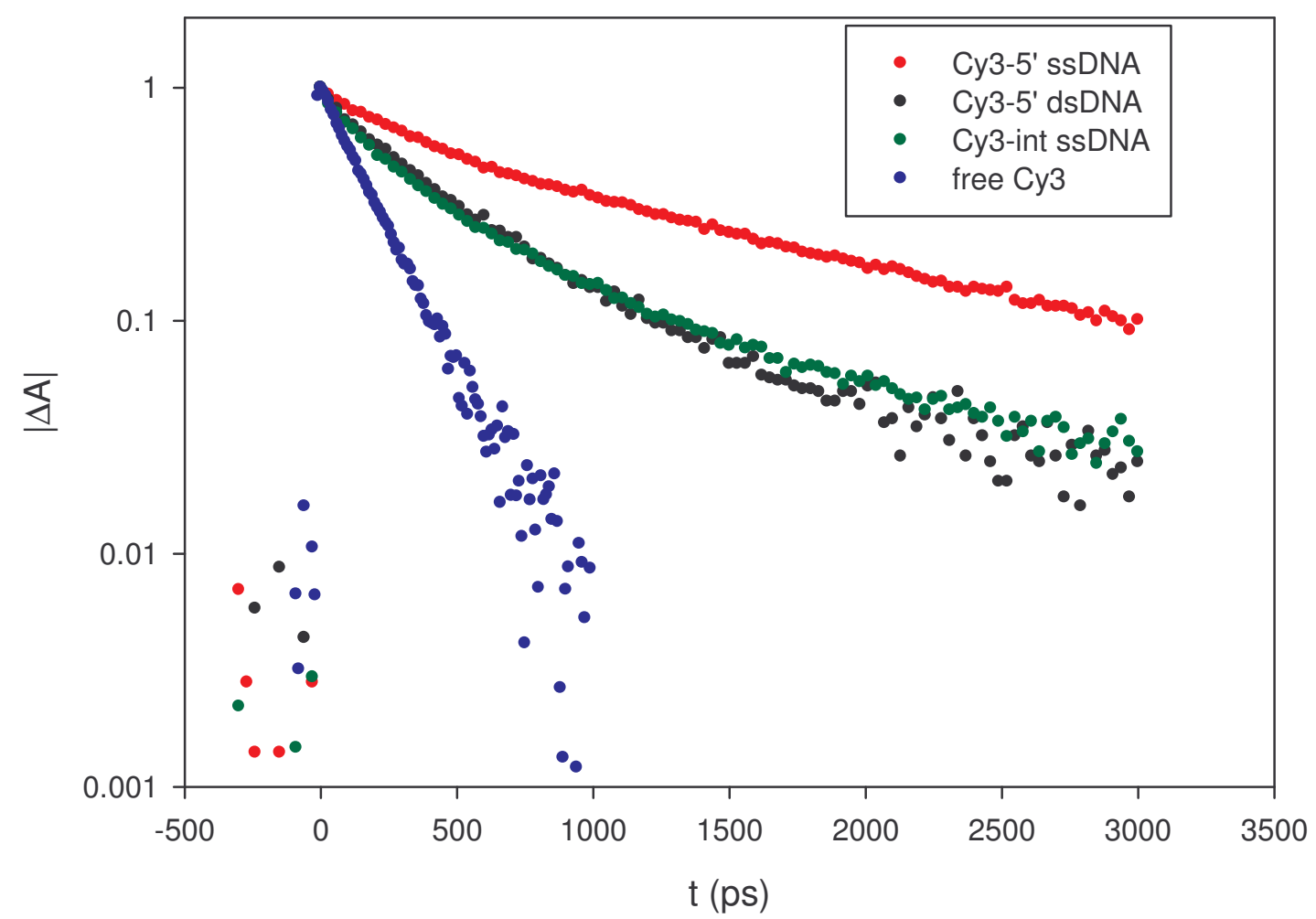

Figure S1: Fluorescence decays as measured by the pump-probe technique. Excitation wavelength: 515nm Excitation pulse width: 150 fs Probe wavelength: 610nm (stimulated emission).

\section{Time-resolved fluorescence anisotropy:}

Figure S2 compares the anisotropy decay of free Cy3 obtained by pump probe (empty circles) and TCSPC (black line). Given the poorer signal-to-noise of the pump-probe experiments, we chose to use the TCSPC results in our quantitative analysis. Both decays were calculated from the measured fluorescence intensities ( $F_{\mathrm{VV}}$ and $\left.F_{\mathrm{VH}}\right)$ without deconvolution. Deconvolution is not needed in pumpprobe, since the pulse width is orders of magnitude smaller than the rotational correlation times being measured. The fact that the decays obtained with both techniques overlap indicates that the lack of deconvolution in TCSP does not introduce experimental artifacts at short times. 


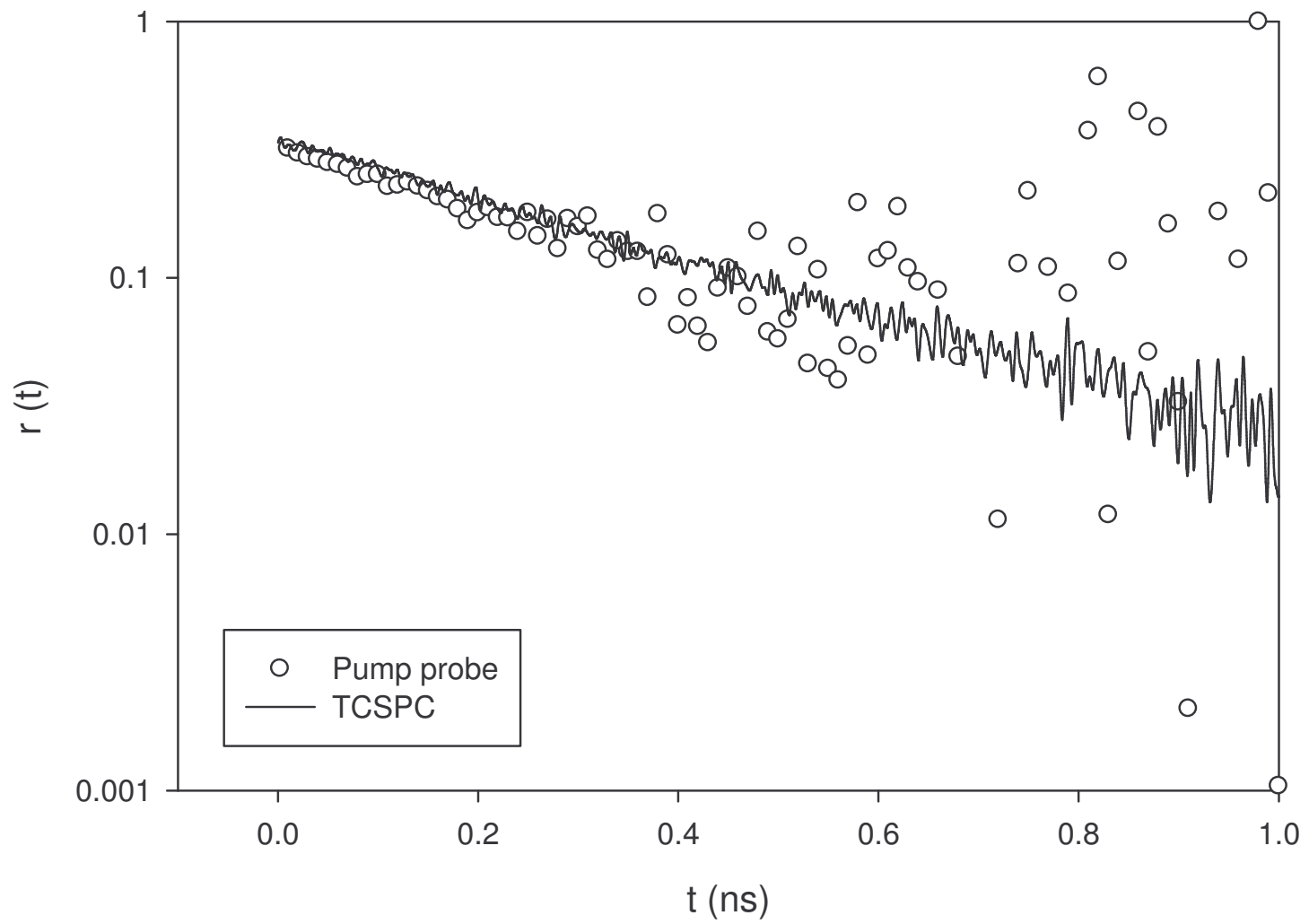

Figure S2: Fluorescence anisotropy decays measured for free Cy3 in aqueous solution. Solid line: decay measured by TCSPC (Excitation pulse width: $\sim 50 \mathrm{ps,} \mathrm{see} \mathrm{manuscript).} \mathrm{Circles:} \mathrm{decay} \mathrm{measured} \mathrm{by}$ pump-probe. (Excitation pulse width: $150 \mathrm{fs}$ )

Further evidence that the short rotational correlation times are not experimental artifacts is provided by the comparison of the TCSPC anisotropy decays with and without deconvolution (figure S3). The inset in figure S3 shows that differences arise only in timescales faster than $\sim 30 \mathrm{ps}$. 


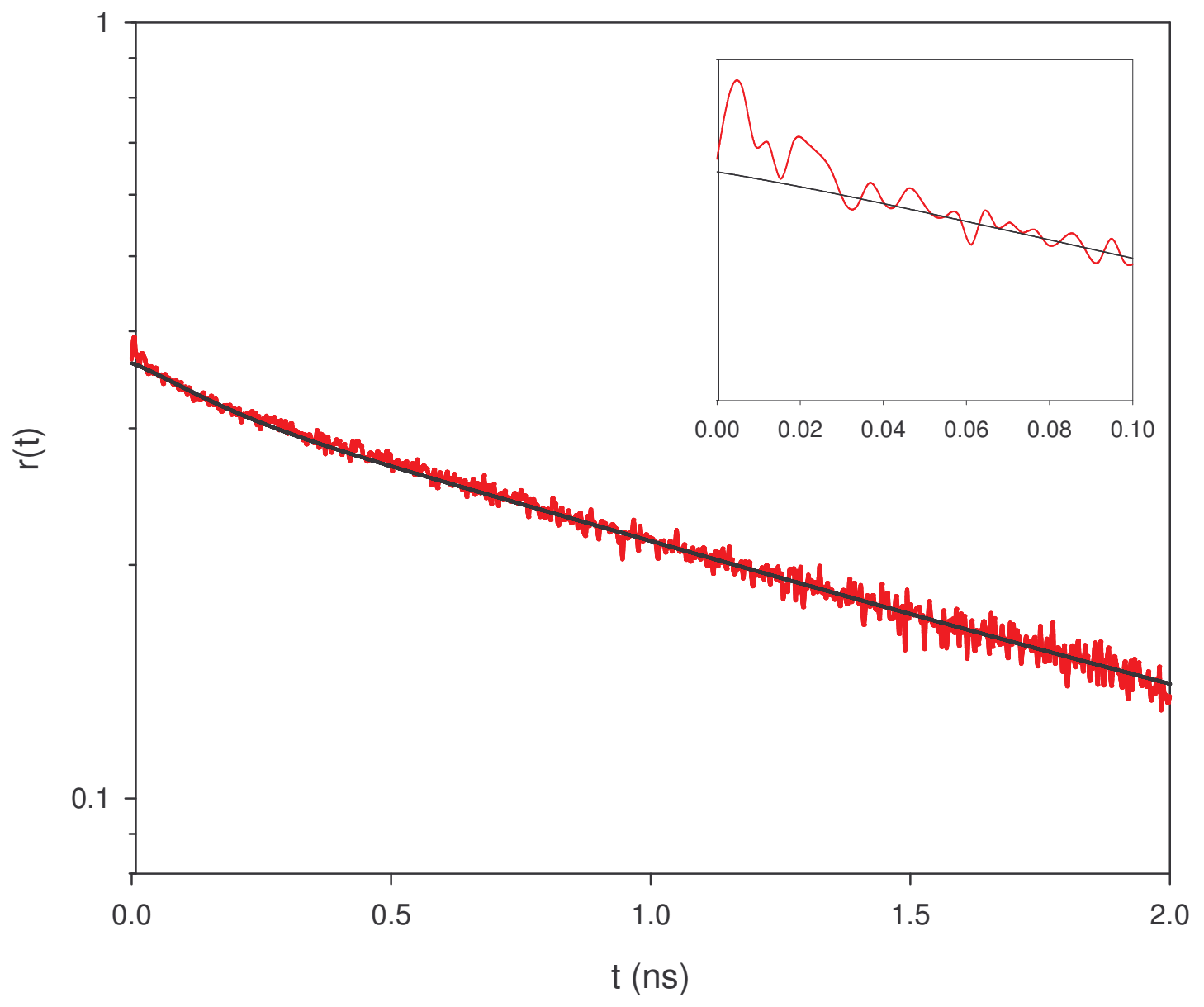

Figure S3: Fluorescence anisotropy decay of Cy3-5' ssDNA. Note that the scale of the ordinate axis is logarithmic. Red line: Anisotropy decay calculated from the experimental intensity decays without deconvolution. Black line: Anisotropy decay obtained by deconvolving $F_{\mathrm{VV}}$ and $F_{\mathrm{VH}}$ using four exponentials as arbitrary fitting functions, and creating $r(t)$ from the resulting functions. Inset: the first 100 ps of the decay

\section{References:}

(1) Causgrove, T. P.; Brune, D. C.; Blankenship, R. E.; Olson, J. M. Photosynthesis Research 1990, $25,1-10$. 